\title{
Activity Coefficients at Infinite Dilution for Organic Compounds Dissolved in 1-Alkyl-1-methylpyrrolidinium Bis(trifluoromethylsulfonyl)imide Ionic Liquids Having Six-, Eight-, and Ten-Carbon Alkyl Chains
}

\author{
William E. Acree, Jr., ${ }^{* \dagger}$ Gary A. Baker, ${ }^{\dagger}$ Anne-Laure Revelli, ${ }^{\S}$ Jean-Charles Moise, ${ }^{\S}$ and Fabrice Mutelet ${ }^{\S}$ \\ ${ }^{\dagger}$ Department of Chemistry, University of North Texas, 1155 Union Circle \#305070, Denton, Texas 76203-5017, United States \\ ${ }^{\ddagger}$ Department of Chemistry, University of Missouri-Columbia, Columbia, Missouri 65211, United States \\ ${ }^{\S}$ Laboratoire de Réactions et Génie des Procédés (UPR CNRS 3349), Université de Lorraine, 1 rue Grandville, BP 2045154001 \\ Nancy, France
}

\begin{abstract}
Activity coefficients at infinite dilution $\left(\gamma_{1,2}^{\infty}\right)$ for 40 diverse probe solutes, including various (cyclo)alkanes, alkenes, alkynes, aromatic hydrocarbons, alcohols, thiophene, ethers, nitroalkanes, and ketones, were measured by inverse gas chromatography at temperatures from 323 to $343 \mathrm{~K}$ in three homologous 1-alkyl-1methylpyrrolidinium bis(trifluoromethylsulfonyl)imide ionic liquids (ILs), bearing hexyl, octyl, and decyl side chains. The retention data were further converted to gasto-IL and water-to-IL partition coefficients using the corresponding gas-to-water partition coefficients. Both sets of partition coefficients were analyzed using the modified Abraham solvation parameter model, with the derived equations tightly correlating the experimental gas-to-IL and water-to-IL partition coefficient data to within average standard deviations of 0.088 and $0.111 \mathrm{log}$ units, respectively.
\end{abstract}

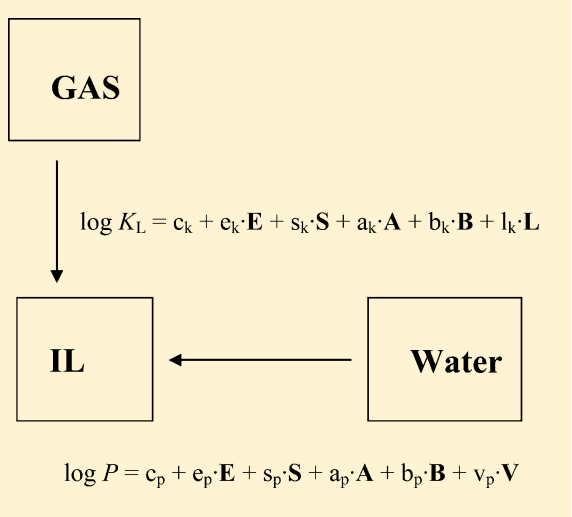

\section{INTRODUCTION}

Ionic liquids (ILs) have been increasingly utilized as solvent media in industrial manufacturing and chemical separation processes because of their low volatility and good thermal stability at high temperatures, recyclability, and thermal nonflammability. Representative applications include the use of ILs as potential green solvents in liquid-liquid extractions involving the removal of aromatic nitrogen and aromatic sulfur compounds from petroleum crude products and fuel oils, ${ }^{1-6}$ as entrainers and azeotrope breakers in extractive distillations, ${ }^{7}$ and as membrane coatings and absorbents for ethylene/ acetylene, ${ }^{8,9}$ propyne/propylene, ${ }^{10}$ propylene/propane, ${ }^{11,12} 1$ hexene/hexane, ${ }^{13}$ and $\mathrm{CO}_{2} / \mathrm{CH}_{4}{ }^{14,15}$ separations. Judicious selection of the cation-anion pair, combined with the introduction of functional groups to the IL, enables one to fine-tune the chemical selectivity and efficiency of the separation process. For example, ether- and hydroxyl-functionalized ILs are reported ${ }^{16}$ to show especially high capacity for solubilizing carbon dioxide and sulfur dioxide.

The present study continues our methodical investigation of the solubilizing ability of IL solvents using the solvation parameter model developed by Abraham and co-workers. ${ }^{17,18}$ The basic model is described by two linear free energy relationships (LFERs). The first relationship involves solute transfer between two condensed phases:

$$
\log P=\mathrm{c}_{\mathrm{p}}+\mathrm{e}_{\mathrm{p}} \cdot \mathbf{E}+\mathrm{s}_{\mathrm{p}} \cdot \mathbf{S}+\mathrm{a}_{\mathrm{p}} \cdot \mathbf{A}+\mathrm{b}_{\mathrm{p}} \cdot \mathbf{B}+\mathrm{v}_{\mathrm{p}} \cdot \mathbf{V}
$$

and the second relationship governs solute transfer from the gas phase to a condensed phase

$$
\log K_{\mathrm{L}}=\mathrm{c}_{\mathrm{k}}+\mathrm{e}_{\mathrm{k}} \cdot \mathbf{E}+\mathrm{s}_{\mathrm{k}} \cdot \mathbf{S}+\mathrm{a}_{\mathrm{k}} \cdot \mathbf{A}+\mathrm{b}_{\mathrm{k}} \cdot \mathbf{B}+\mathrm{l}_{\mathrm{k}} \cdot \mathbf{L}
$$

where $P$ and $K_{\mathrm{L}}$ refer to the solute's condensed phase-tocondensed phase partition coefficient (often the water-toorganic solvent partition coefficient) and the gas-to-condensed phase partition coefficient, respectively. The uppercase letters in eqs 1 and 2 represent the solute properties, whereas the lowercase letters represent the complementary properties of the ILs. The solute descriptors are the excess molar refraction (E), the combined dipolarity/polarizability (S), the hydrogen-bond acidity (A) and basicity (B), the logarithm of the solute's gasto- $n$-hexadecane partition coefficient at $298 \mathrm{~K}(\mathrm{~L})$, and the solute's McGowan volume in units of $\left(\mathrm{cm}^{3} \mathrm{~mol}^{-1}\right) / 100(\mathrm{~V})$. Solute descriptors have been reported for about 5000 solutes based on experimental partition coefficient and chromatographic retention factor data or calculated by the group contribution method. ${ }^{17-24}$

The advantage of characterizing ILs with the Abraham model is that once the equation coefficients are calculated, one can estimate $\log P$ and $\log K_{\mathrm{L}}$ values of additional solutes provided that the solute coefficients are known. To date, we have

Received: June 25, 2012

Accepted: October 17, 2012

Published: October 31, 2012 
characterized the solubilizing properties of more than 30 monocationic $\mathrm{ILs}^{25-36}$ and six trigeminal tricationic $\mathrm{ILs}^{37}$ using eqs 1 and 2 of the solvation parameter model. We have also extended the solvation parameter model to include cation- and anion-specific equation coefficients by splitting each equation coefficient into distinct cation and anion contributions (i.e., $c_{\mathrm{k}}=$ $c_{\mathrm{k}, \mathrm{cation}}+c_{\mathrm{k}, \text { anion }}$, and so forth), ${ }^{38-41}$ and to permit the estimation $\log K_{\mathrm{L}}$ and $\log P$ values for solutes dissolved in anhydrous ILs based on calculated temperature-dependent IL functional group parameters for 21 groups: 12 groups characterizing the cations and 9 groups for the anions. ${ }^{42}$ Previous studies have shown that the best $\log K_{\mathrm{L}}$ and $\log P$ predictions are obtained with ILspecific correlations (eqs 1 and 2), as one might expect. Studies have further shown that the predictive ability of the ion-specific equations is slightly better than expressions based on the group contribution version of the model. For many IL solvents, however, the group contribution model will be the only option available.

Although substantial progress has been made, the 24 different cation-specific and 14 different anion-specific equation coefficients and the 21 different IL functional group parameters that have been determined thus far do not allow us to estimate the solubilizing behavior of all ILs that are currently being tested or employed in industrial manufacturing or of interest in chemical separation processes. To expand the number of predictive correlations we have measured infinite dilution activity coefficients and gas-to-liquid partition coefficients for a series of organic compounds dissolved in the 1-alkyl-1methylpyrrolidinium bis(trifluoromethylsulfonyl)imide IL homologues 1-hexyl-1-methylpyrrolidinium bis(trifluoromethylsulfonyl)imide ([HMPyrr $\left.]^{+}\left[\mathrm{Tf}_{2} \mathrm{~N}\right]^{-}\right)$, 1-octyl-1-methylpyrrolidinium bis(trifluoromethylsulfonyl)imide ([OMPyrr $\left.]^{+}\left[\mathrm{Tf}_{2} \mathrm{~N}\right]^{-}\right)$, and 1-decyl-1-methylpyrrolidinium bis(trifluoromethylsulfonyl)imide $\left([\mathrm{DMPyrr}]^{+}\left[\mathrm{Tf}_{2} \mathrm{~N}\right]^{-}\right)$. These experimental data are used to derive IL-specific Abraham model $\log K_{\mathrm{L}}$ and $\log P$ correlations and to calculate ion-specific equation coefficients for the $[\mathrm{HMPyrr}]^{+},[\mathrm{OMPyrr}]^{+}$, and $[\mathrm{DMPyrr}]^{+}$cations.

\section{EXPERIMENTAL PROCEDURES AND RESULTS}

Materials or Chemicals. Synthesis and characterization of the 1-alkyl-1-methylpyrrolidinium bis(trifluoromethylsulfonyl)imide ILs was performed as described earlier by Jin et al. ${ }^{43}$ with special attention paid to procedures and precautions necessary for attaining highly pure "spec-grade" material. ${ }^{44,45}$

Apparatus and Experimental Procedure. Inverse chromatography experiments were performed using a Varian CP-3800 gas chromatograph equipped with a heated oncolumn injector and a flame ionization detector. The injector and detector temperatures were maintained at $523 \mathrm{~K}$ during all experimental measurements. Helium flow rate was adjusted to obtain adequate retention times. Methane was used to determine the column hold-up time. Exit gas flow rates were determined using an Alltech Digital Flow Check Mass Flowmeter. The temperature of the oven was measured with a Pt 100 probe and controlled to within $0.1 \mathrm{~K}$. A personal computer directly recorded detector signals and the corresponding chromatograms were constructed using Galaxie software.

Packed columns of 1-m length containing from 15 to $35 \%$ IL stationary phase on Chromosorb WHP (60-80 mesh) were prepared using the rotary evaporator technique. After evaporation of the chloroform under vacuum, the support was equilibrated at $333 \mathrm{~K}$ over the course of $6 \mathrm{~h}$. Prior to chromatographic measurement, each packed column was conditioned for $12 \mathrm{~h}$ at $363 \mathrm{~K}$ with a flow rate of $20 \mathrm{~cm}^{3}$ $\mathrm{min}^{-1}$. The mass of the packing material was calculated from the mass of the packed and empty columns and was checked throughout experiments. The masses of the stationary phase were determined with a precision of $0.0003 \mathrm{~g}$. A $1-5 \mu \mathrm{L}$ volume of the headspace vapor of samples was introduced and validated to be within infinite dilution conditions. Each experiment was repeated at least twice to check reproducibility. Retention times were generally reproducible to within 0.01 to $0.03 \mathrm{~min}$. The retention time measurements were repeated systematically each day for three selected solutes in order to certify the day-to-day stability of the experimental setup and to rule out possible drift caused by elution of the stationary phase by the helium stream. No changes in the retention times were noted throughout the study.

Theoretical Basis. The retention data determined from inverse chromatography experiments were used to calculate partition coefficients of the solutes in the IL of interest. The net retention volume $\left(\mathrm{V}_{\mathrm{N}}\right)$ was calculated by the following usual relationship ${ }^{25}$

$$
V_{\mathrm{N}}=\frac{2}{3} \frac{\left[\left(\frac{P_{\mathrm{i}}}{P_{\mathrm{o}}}\right)^{2}-1\right]}{\left[\left(\frac{P_{\mathrm{i}}}{P_{\mathrm{o}}}\right)^{3}-1\right]} U_{0} t_{\mathrm{R}}^{\prime} \frac{T_{\mathrm{col}}}{T_{\mathrm{r}}}\left(1-\frac{P_{0 \mathrm{~W}}}{P_{0}}\right)
$$

The adjusted retention time $\left(t_{\mathrm{R}}^{\prime}\right)$ was computed as a difference between the retention time of a solute and that of methane, $T_{\mathrm{col}}$ is the column temperature, $U_{0}$ is the flow rate of the carrier gas measured at room temperature $\left(T_{\mathrm{r}}\right)$, and $P_{\mathrm{ow}}$ is the vapor pressure of water at $T_{\mathrm{r}} . P_{\mathrm{i}}$ and $P_{\mathrm{o}}$ are respectively the inlet and outlet pressures.

The activity coefficient at infinite dilution of solute 1 in IL 2 $\left(\gamma_{1,2}^{\infty}\right)$ is calculated from the following expression: ${ }^{46}$

$$
\ln \gamma_{1,2}^{\infty}=\ln \left(\frac{n_{2} R T}{V_{\mathrm{N}} P_{1}^{0}}\right)-P_{1}^{0} \frac{B_{11}-V_{1}^{0}}{R T}+\frac{2 B_{13}-V_{1}^{\infty}}{R T} J P_{\mathrm{o}}
$$

Here $n_{2}$ is the mole number of the stationary phase component inside the column, $R$ is the ideal gas constant, $T$ denotes the temperature of the oven, $B_{11}$ refers to the second virial coefficient of the solute in the gaseous state at temperature $T$, $B_{13}$ represents the mutual virial coefficient between the solute 1 and the carrier gas helium (denoted 3 ), and $P_{1}^{0}$ is the probe vapor pressure at temperature $T$.

Partition coefficient $\left(K_{\mathrm{L}}\right)$ may at this juncture be calculated from the activity coefficients at infinite dilution $\left(\gamma_{1,2}^{\infty}\right)$ using the following expression:

$$
K_{\mathrm{L}}=\frac{R T}{\gamma_{1,2}^{\infty} P_{1}^{0} V_{\text {solvent }}}
$$

Physical and thermodynamic properties of pure solutes needed for these calculations are given in a previous work. ${ }^{28}$

\section{RESULTS AND DISCUSSION}

Activity Coefficients and Selectivity at Infinite Dilution. The error in the experimental determination of activity coefficients is evaluated at about $3 \%$. For all ILs studied in this work, no interfacial adsorption was observed, and the average relative standard deviation between data sets obtained 
from two different packed columns was just 3-4\%. Experimental activity coefficients at infinite dilution and gasto-IL partition coefficients calculated using eqs 3-7 are listed in Tables 1-6.

Table 1. Infinite Dilution Activity Coefficients and Partition Coefficients $\log K_{\mathrm{L}}$ and $\log P$ of Organic Solutes in $[\text { HMPyrr }]^{+}\left[\mathrm{Tf}_{2} \mathbf{N}\right]^{-}$

\begin{tabular}{|c|c|c|c|c|c|}
\hline \multirow[b]{2}{*}{ solutes } & \multicolumn{3}{|c|}{$\gamma^{\infty}$ at $T / \mathrm{K}$} & $\log K_{\mathrm{L}}$ & $\log P$ \\
\hline & 322.9 & 333.0 & 343.1 & \multicolumn{2}{|c|}{298.2} \\
\hline hexane & 7.74 & 7.38 & 7.11 & 1.52 & 3.34 \\
\hline 3-methylpentane & 6.52 & 6.46 & 6.37 & 1.53 & 3.37 \\
\hline heptane & 10.58 & 9.43 & 9.58 & 1.90 & 3.86 \\
\hline octane & 14.25 & 13.37 & 12.91 & 2.27 & 4.38 \\
\hline nonane & 21.10 & 18.18 & 18.49 & 2.65 & 4.80 \\
\hline decane & 25.99 & 18.97 & 22.74 & 3.03 & 5.35 \\
\hline undecane & 34.39 & 30.96 & 30.11 & 3.39 & 5.77 \\
\hline methylcyclopentane & 4.90 & 4.52 & 4.76 & 1.80 & 2.97 \\
\hline cyclohexane & 5.16 & 4.64 & 4.67 & 1.88 & 2.78 \\
\hline methylcyclohexane & 6.57 & 6.07 & 6.03 & 2.11 & 3.36 \\
\hline cycloheptane & 14.01 & 19.01 & 25.09 & 2.39 & 2.98 \\
\hline benzene & 0.76 & 0.77 & 0.78 & 2.80 & 2.17 \\
\hline toluene & 1.00 & 1.01 & 1.04 & 3.20 & 2.55 \\
\hline ethylbenzene & 1.38 & 1.43 & 1.46 & 3.53 & 2.95 \\
\hline m-xylene & 1.28 & 1.39 & 1.42 & 3.66 & 3.05 \\
\hline p-xylene & 1.35 & 1.40 & 1.42 & 3.60 & 3.01 \\
\hline o-xylene & 1.17 & 1.28 & 1.31 & 3.79 & 3.05 \\
\hline 1-hexene & 4.56 & 4.63 & 4.44 & 1.69 & 2.85 \\
\hline 1-hexyne & 2.10 & 2.10 & 2.09 & 2.22 & 2.43 \\
\hline 1-heptyne & 2.24 & 2.79 & 2.78 & 2.80 & 3.24 \\
\hline 2-butanone & 0.42 & 0.42 & 0.39 & 3.08 & 0.36 \\
\hline 2-pentanone & 0.59 & 0.65 & 0.66 & 3.39 & 0.81 \\
\hline 3-pentanone & 0.47 & 0.60 & 0.62 & 3.53 & 1.03 \\
\hline 1,4-dioxane & 0.58 & 0.42 & 0.44 & 3.18 & -0.53 \\
\hline ethanol & 1.41 & 1.40 & 1.31 & 2.67 & -1.00 \\
\hline 1-propanol & 1.72 & 1.64 & 1.49 & 2.98 & -0.58 \\
\hline 2-propanol & 1.55 & 1.53 & 1.40 & 2.73 & -0.75 \\
\hline diethyl ether & 1.56 & 1.76 & 1.76 & 1.79 & 0.62 \\
\hline diisopropyl ether & 3.18 & 3.56 & 3.53 & 2.02 & 0.97 \\
\hline chloroform & 0.68 & 0.72 & 0.74 & 2.57 & 1.78 \\
\hline dichloromethane & 0.31 & 0.36 & 0.35 & 2.44 & 1.48 \\
\hline tetrachloromethane & 1.78 & 1.85 & 1.86 & 2.35 & 2.54 \\
\hline acetonitrile & 0.46 & 0.46 & 0.48 & 3.05 & 0.20 \\
\hline nitromethane & 0.57 & 0.57 & 0.56 & 3.32 & 0.37 \\
\hline 1-nitropropane & 0.66 & 0.66 & 0.65 & 3.81 & 1.36 \\
\hline pyridine & 0.52 & 0.52 & 0.53 & 3.62 & 0.18 \\
\hline thiophene & 0.70 & 0.71 & 0.72 & 2.91 & 1.87 \\
\hline
\end{tabular}

Activity coefficients at infinite dilution for most organic compounds were found to decrease with increased temperature. As observed with imidazolium and ammonium cations, the solubility of apolar compounds increases with an increase in the alkyl chain length grafted onto the pyrrolidinium cation. For compounds containing the same number of carbon atoms but originating from different solute families, the trend $\gamma_{\text {aromatic }}<$ $\gamma_{\text {alkyne }}<\gamma_{\text {alkene }}<\gamma_{\text {alkane }}$ was observed. This overall trend is followed for all ILs, regardless of the cation or anion identity. That is to say, the presence of double or triple bonds in a solute tends to increases the solute/IL interactions.

For the alcohols, the low $\gamma_{1,2}^{\infty}$ values can be ascribed to the lone pair of electrons on the oxygen atom of the alcohol interacting with the positively charged cation and to the acidic
Table 2. Logarithm of Partition Coefficients $\log K_{\mathrm{L}}$ of Organic Solutes in $[\mathrm{HMPyrr}]^{+}\left[\mathrm{Tf}_{2} \mathrm{~N}\right]^{-}$

\begin{tabular}{|c|c|c|c|c|c|}
\hline \multirow[b]{2}{*}{ solutes } & \multicolumn{5}{|c|}{$T / \mathrm{K}$} \\
\hline & 322.9 & 333.0 & 343.1 & $R^{2}$ & 298.2 \\
\hline hexane & 1.20 & 1.08 & 0.97 & 1.000 & 1.52 \\
\hline 3-methylpentane & 1.19 & 1.06 & 0.95 & 0.999 & 1.53 \\
\hline heptane & 1.52 & 1.41 & 1.26 & 0.991 & 1.90 \\
\hline octane & 1.84 & 1.69 & 1.54 & 1.000 & 2.27 \\
\hline nonane & 2.16 & 2.01 & 1.81 & 0.991 & 2.65 \\
\hline decane & 2.47 & 2.38 & 2.09 & 0.918 & 3.03 \\
\hline undecane & 2.79 & 2.58 & 2.36 & 1.000 & 3.39 \\
\hline methylcyclopentane & 1.44 & 1.34 & 1.19 & 0.988 & 1.80 \\
\hline cyclohexane & 1.55 & 1.45 & 1.31 & 0.991 & 1.88 \\
\hline methylcyclohexane & 1.74 & 1.62 & 1.48 & 0.998 & 2.11 \\
\hline cycloheptane & 2.02 & 1.88 & 1.75 & 0.999 & 2.39 \\
\hline benzene & 2.38 & 2.23 & 2.09 & 0.999 & 2.80 \\
\hline toluene & 2.73 & 2.55 & 2.39 & 1.000 & 3.20 \\
\hline ethylbenzene & 3.00 & 2.80 & 2.63 & 0.999 & 3.53 \\
\hline m-xylene & 3.09 & 2.87 & 2.69 & 0.996 & 3.66 \\
\hline p-xylene & 3.06 & 2.85 & 2.67 & 0.999 & 3.60 \\
\hline o-xylene & 3.22 & 2.99 & 2.81 & 0.996 & 3.79 \\
\hline 1-hexene & 1.35 & 1.21 & 1.10 & 0.993 & 1.69 \\
\hline 1-hexyne & 1.82 & 1.67 & 1.54 & 0.999 & 2.22 \\
\hline 1-heptyne & 2.24 & 1.98 & 1.83 & 0.977 & 2.80 \\
\hline 2-butanone & 2.60 & 2.41 & 2.25 & 0.995 & 3.08 \\
\hline 2-pentanone & 2.88 & 2.67 & 2.50 & 0.995 & 3.39 \\
\hline 3-pentanone & 2.94 & 2.66 & 2.49 & 0.980 & 3.53 \\
\hline 1,4-dioxane & 2.86 & 2.83 & 2.65 & 0.874 & 3.18 \\
\hline ethanol & 2.20 & 2.02 & 1.87 & 0.995 & 2.67 \\
\hline 1-propanol & 2.50 & 2.31 & 2.15 & 0.997 & 2.98 \\
\hline 2-propanol & 2.26 & 2.06 & 1.92 & 0.993 & 2.73 \\
\hline diethyl ether & 1.40 & 1.22 & 1.11 & 0.984 & 1.79 \\
\hline diisopropyl ether & 1.59 & 1.40 & 1.27 & 0.987 & 2.02 \\
\hline chloroform & 2.15 & 1.99 & 1.85 & 0.998 & 2.57 \\
\hline dichloromethane & 2.08 & 1.90 & 1.82 & 0.960 & 2.44 \\
\hline tetrachloromethane & 1.95 & 1.79 & 1.66 & 0.997 & 2.35 \\
\hline acetonitrile & 2.62 & 2.48 & 2.32 & 0.999 & 3.05 \\
\hline nitromethane & 2.87 & 2.70 & 2.55 & 0.999 & 3.32 \\
\hline 1-nitropropane & 3.30 & 3.11 & 2.93 & 0.999 & 3.81 \\
\hline pyridine & 3.13 & 2.95 & 2.78 & 1.000 & 3.62 \\
\hline thiophene & 2.49 & 2.33 & 2.18 & 0.999 & 2.91 \\
\hline
\end{tabular}

proton of the alcohol hydrogen bonding with oxygen atoms of the anion. Branched-chain alcohols have a lower solubility compared to linear alcohols, and their solubility decreases with increasing chain length. Notably, ketones strongly interact with these ILs, showing even higher solubility. Pyridine and nitroalkanes have strong interactions with all three ILs.

Table 7 compares values of $\gamma^{\infty}$ in $[\mathrm{HMPyrr}]^{+}\left[\mathrm{Tf}_{2} \mathrm{~N}\right]^{-}$and $[\mathrm{OMPyrr}]^{+}\left[\mathrm{Tf}_{2} \mathrm{~N}\right]^{-}$obtained in this work with $\gamma^{\infty}$ measured by Nebig et al. ${ }^{47}$ at $T=323.15 \mathrm{~K}$. In some cases, the differences are outstandingly small (for example, $0.2 \%$ for 1-hexene in $\left.[\mathrm{HMPyrr}]^{+}\left[\mathrm{Tf}_{2} \mathrm{~N}\right]^{-}\right)$, but the overall standard deviation for these solutes is around $5 \%$, which is within expectations when comparing dilutor technique results to inverse gas chromatography measurements.

The measurements conducted during this research offer an important means to evaluate the performance of pyrrolidiniumbased ILs as solvents in various separation problems. This is achieved through the calculation of selectivity $\left(S_{12}^{\infty}\right)$ and capacity $\left(k_{1}^{\infty}\right)$ values at infinite dilution 
Table 3. Infinite Dilution Activity Coefficients and Partition Coefficients $\log K_{\mathrm{L}}$ and $\log P$ of Organic Solutes in $[\mathrm{OMPyrr}]^{+}\left[\mathrm{Tf}_{2} \mathrm{~N}\right]^{-}$

\begin{tabular}{|c|c|c|c|c|c|}
\hline \multirow[b]{2}{*}{ solutes } & \multicolumn{3}{|c|}{$\gamma^{\infty}$ at $T / K$} & $\log K_{\mathrm{L}}$ & $\log P$ \\
\hline & 323.0 & 333.0 & 343.1 & \multicolumn{2}{|c|}{298.2} \\
\hline hexane & 5.01 & 4.80 & 4.05 & 1.63 & 3.45 \\
\hline 3-methylpentane & 4.61 & 4.52 & 4.08 & 1.63 & 3.47 \\
\hline heptane & 6.40 & 6.06 & 5.13 & 2.03 & 3.99 \\
\hline octane & 8.10 & 7.80 & 6.54 & 2.44 & 4.55 \\
\hline nonane & 11.27 & 10.72 & 9.14 & 2.86 & 5.01 \\
\hline decane & 14.77 & 12.51 & 10.98 & 3.15 & 5.47 \\
\hline undecane & 16.82 & 15.64 & 14.64 & 3.70 & 6.08 \\
\hline methylcyclopentane & 3.13 & 3.36 & 2.84 & 1.94 & 3.11 \\
\hline cyclohexane & 3.45 & 3.31 & 3.06 & 2.04 & 2.94 \\
\hline methylcyclohexane & 4.22 & 4.04 & 3.90 & 2.31 & 3.56 \\
\hline cycloheptane & 8.62 & 12.10 & 15.39 & 2.60 & 3.19 \\
\hline benzene & 0.60 & 0.62 & 0.61 & 2.89 & 2.26 \\
\hline toluene & 0.76 & 0.78 & 0.74 & 3.27 & 2.62 \\
\hline ethylbenzene & 1.03 & 0.98 & 1.04 & 3.64 & 3.06 \\
\hline m-xylene & 1.00 & 0.84 & 1.05 & 3.77 & 3.16 \\
\hline p-xylene & 1.01 & 0.99 & 1.05 & 3.72 & 3.13 \\
\hline o-xylene & 0.92 & 0.87 & 0.97 & 3.88 & 3.14 \\
\hline 1-hexene & 3.17 & 3.02 & 2.58 & 1.74 & 2.90 \\
\hline 1-hexyne & 1.57 & 1.58 & 1.34 & 2.25 & 2.46 \\
\hline 1-heptyne & 1.94 & 1.89 & 1.90 & 2.74 & 3.18 \\
\hline 2-butanone & 0.36 & 0.34 & 0.31 & 3.11 & 0.39 \\
\hline 2-pentanone & 0.49 & 0.51 & 0.48 & 3.40 & 0.82 \\
\hline 3-pentanone & 0.45 & 0.47 & 0.44 & 3.38 & 0.88 \\
\hline 1,4-dioxane & 0.32 & 0.60 & 0.31 & 3.47 & -0.24 \\
\hline methanol & 0.97 & 1.09 & 0.71 & 2.33 & -1.41 \\
\hline ethanol & 1.33 & 1.26 & 0.98 & 2.55 & -1.12 \\
\hline 1-propanol & 1.49 & 1.29 & 1.14 & 2.99 & -0.57 \\
\hline 2-propanol & 1.40 & 1.27 & 1.06 & 2.67 & -0.81 \\
\hline 2-methyl-1-propanol & 1.59 & 1.44 & 1.22 & 3.20 & -0.10 \\
\hline diethyl ether & 1.34 & 1.34 & 1.14 & 1.69 & 0.52 \\
\hline diisopropyl ether & 2.57 & 2.58 & 2.26 & 1.98 & 0.93 \\
\hline chloroform & 0.56 & 0.58 & 0.53 & 2.56 & 1.77 \\
\hline dichloromethane & 0.26 & 0.27 & 0.28 & 2.51 & 1.55 \\
\hline tetrachloromethane & 1.39 & 1.30 & 1.15 & 2.33 & 2.52 \\
\hline acetonitrile & 0.43 & 0.39 & 0.37 & 2.98 & 0.13 \\
\hline nitromethane & 0.54 & 0.51 & 0.48 & 3.29 & 0.34 \\
\hline 1-nitropropane & 0.55 & 0.52 & 0.51 & 3.85 & 1.40 \\
\hline pyridine & 0.42 & 0.39 & 0.41 & 3.68 & 0.24 \\
\hline thiophene & 0.57 & 0.55 & 0.53 & 2.97 & 1.93 \\
\hline
\end{tabular}

$$
\begin{aligned}
& S_{12}^{\infty}=\frac{\gamma_{1 / \mathrm{RTIL}}^{\infty}}{\gamma_{2 / \mathrm{RTIL}}^{\infty}} \\
& k_{1}^{\infty}=\frac{1}{\gamma_{1 / \mathrm{RTIL}}^{\infty}}
\end{aligned}
$$

In eq $6, \gamma_{1 / \text { RTIL }}^{\infty}$ and $\gamma_{2 / \text { RTIL }}^{\infty}$ denote the infinite dilution coefficients of solutes 1 and 2, respectively, in the IL solvent. Selectivities and capacities at infinite dilution are collected in Table 8 for four separation problems at $323.15 \mathrm{~K}$; these include hexane/benzene, hexane/methanol, hexane/thiophene, and cyclohexane/thiophene separations. With regard to aliphatic/ aromatic separations, these results indicate that pyrrolidiniumbased ILs generally exhibit poorer performances compared to imidazolium-based ILs. The selectivities obtained using pyrrolidinium-based ILs (between 6 and 10) are lower than that for classical solvents used industrially such as sulfolane
Table 4. Logarithm of Partition Coefficients $\log K_{\mathrm{L}}$ of

\begin{tabular}{|c|c|c|c|c|c|}
\hline \multirow[b]{2}{*}{ solutes } & \multicolumn{5}{|c|}{$T / \mathrm{K}$} \\
\hline & 323.0 & 333.0 & 343.1 & $R^{2}$ & 298.2 \\
\hline hexane & 1.40 & 1.28 & 1.23 & 0.956 & 1.63 \\
\hline 3-methylpentane & 1.35 & 1.23 & 1.15 & 0.984 & 1.63 \\
\hline heptane & 1.75 & 1.61 & 1.54 & 0.974 & 2.03 \\
\hline octane & 2.10 & 1.93 & 1.84 & 0.973 & 2.44 \\
\hline nonane & 2.44 & 2.25 & 2.13 & 0.987 & 2.86 \\
\hline decane & 2.72 & 2.57 & 2.42 & 1.000 & 3.15 \\
\hline undecane & 3.11 & 2.89 & 2.68 & 1.000 & 3.70 \\
\hline methylcyclopentane & 1.65 & 1.48 & 1.42 & 0.921 & 1.94 \\
\hline cyclohexane & 1.73 & 1.61 & 1.51 & 0.996 & 2.04 \\
\hline methylcyclohexane & 1.94 & 1.81 & 1.68 & 1.000 & 2.31 \\
\hline cycloheptane & 2.24 & 2.09 & 1.97 & 0.991 & 2.60 \\
\hline benzene & 2.49 & 2.34 & 2.20 & 0.998 & 2.89 \\
\hline toluene & 2.86 & 2.68 & 2.55 & 0.992 & 3.27 \\
\hline ethylbenzene & 3.14 & 2.98 & 2.78 & 0.996 & 3.64 \\
\hline m-xylene & 3.21 & 3.10 & 2.83 & 0.949 & 3.77 \\
\hline p-xylene & 3.19 & 3.01 & 2.81 & 0.999 & 3.72 \\
\hline o-xylene & 3.34 & 3.17 & 2.95 & 0.993 & 3.88 \\
\hline 1-hexene & 1.52 & 1.41 & 1.35 & 0.965 & 1.74 \\
\hline 1-hexyne & 1.96 & 1.81 & 1.74 & 0.950 & 2.25 \\
\hline 1-heptyne & 2.31 & 2.16 & 2.00 & 1.000 & 2.74 \\
\hline 2-butanone & 2.67 & 2.51 & 2.36 & 0.999 & 3.11 \\
\hline 2-pentanone & 2.96 & 2.78 & 2.65 & 0.993 & 3.40 \\
\hline 3-pentanone & 2.96 & 2.78 & 2.65 & 0.992 & 3.38 \\
\hline 1,4-dioxane & 3.12 & 2.69 & 2.81 & 0.489 & 3.47 \\
\hline methanol & 2.10 & 1.88 & 1.91 & 0.640 & 2.33 \\
\hline ethanol & 2.24 & 2.08 & 2.01 & 0.947 & 2.55 \\
\hline 1-propanol & 2.57 & 2.43 & 2.28 & 1.000 & 2.99 \\
\hline 2-propanol & 2.31 & 2.15 & 2.05 & 0.986 & 2.67 \\
\hline 2-methyl-1-propanol & 2.76 & 2.58 & 2.45 & 0.994 & 3.20 \\
\hline diethyl ether & 1.47 & 1.35 & 1.31 & 0.932 & 1.69 \\
\hline diisopropyl ether & 1.69 & 1.55 & 1.48 & 0.968 & 1.98 \\
\hline chloroform & 2.24 & 2.09 & 2.01 & 0.974 & 2.56 \\
\hline dichloromethane & 2.17 & 2.03 & 1.92 & 0.998 & 2.51 \\
\hline tetrachloromethane & 2.07 & 1.96 & 1.88 & 0.989 & 2.33 \\
\hline acetonitrile & 2.67 & 2.56 & 2.44 & 0.998 & 2.98 \\
\hline nitromethane & 2.91 & 2.76 & 2.63 & 0.997 & 3.29 \\
\hline 1-nitropropane & 3.38 & 3.22 & 3.05 & 1.000 & 3.85 \\
\hline pyridine & 3.22 & 3.08 & 2.90 & 0.995 & 3.68 \\
\hline thiophene & 2.59 & 2.49 & 2.32 & 0.973 & 2.97 \\
\hline
\end{tabular}
Organic Solutes in $[\mathrm{OMPyrr}]^{+}\left[\mathrm{Tf}_{2} \mathrm{~N}\right]^{-}$

$\left(S_{\text {hexane/benzene }}=30.5\right)$, dimethylsulfoxide $\left(S_{\text {hexane/benzene }}=22.7\right)$, and $N$-methyl-2-pyrrolidinone $\left(S_{\text {hexane/benzene }}=12.5\right) .{ }^{38}$ All the same, a better capacity and selectivity may be obtained for relatively short alkyl chains grafted to the pyrrolidinium cation, as observed with 1-butyl-1-methylpyrrolidinium. ${ }^{48}$ Indeed, long alkyl chains grafted onto the cation are known to increase capacity, while being a detriment to selectivity. For desulfurization applications, pyrrolidinium-based ILs show a large capacity for thiophene and selectivity is relatively high as well.

Linear Solvation Energy Relationship (LSER) Characterization. The Abraham solvation parameter model provides a very convenient method to mathematically describe the solubilizing properties of IL solvents. Mathematical correlations of the type of eqs 1 and 2 can be developed for each individual $\mathrm{IL}$, or alternatively, one may use the ion-specific version of the model suggested by Sprunger et al. ${ }^{38-41}$ 
Table 5. Infinite Dilution Activity Coefficients and Partition Coefficients $\log K_{\mathrm{L}}$ and $\log P$ of Organic Solutes in $[\mathrm{DMPyrr}]^{+}\left[\mathrm{Tf}_{2} \mathbf{N}\right]^{-}$

\begin{tabular}{|c|c|c|c|c|c|}
\hline \multirow[b]{2}{*}{ solutes } & \multicolumn{3}{|c|}{$\gamma^{\infty}$ at $T / K$} & $\log K_{\mathrm{L}}$ & $\log P$ \\
\hline & 322.6 & 332.5 & 342.8 & \multicolumn{2}{|c|}{298.2} \\
\hline hexane & 3.97 & 3.78 & 3.68 & 1.81 & 3.63 \\
\hline 3-methylpentane & 3.65 & 3.36 & 3.40 & 1.76 & 3.60 \\
\hline heptane & 4.91 & 4.75 & 4.62 & 2.24 & 4.20 \\
\hline octane & 6.13 & 5.92 & 5.76 & 2.66 & 4.77 \\
\hline nonane & 8.32 & 8.01 & 7.65 & 3.06 & 5.21 \\
\hline decane & 9.42 & 9.05 & 8.70 & 3.47 & 5.79 \\
\hline undecane & 11.68 & 11.18 & 10.66 & 3.88 & 6.26 \\
\hline methylcyclopentane & 2.90 & 2.76 & 2.66 & 1.98 & 3.15 \\
\hline cyclohexane & 2.81 & 2.71 & 2.62 & 2.15 & 3.05 \\
\hline methylcyclohexane & 3.38 & 3.28 & 3.15 & 2.40 & 3.65 \\
\hline cycloheptane & 7.00 & 9.72 & 13.35 & 2.71 & 3.30 \\
\hline benzene & 0.62 & 0.63 & 0.64 & 2.88 & 2.25 \\
\hline toluene & 0.77 & 0.78 & 0.80 & 3.31 & 2.66 \\
\hline ethylbenzene & 1.01 & 1.04 & 1.06 & 3.65 & 3.07 \\
\hline m-xylene & 0.99 & 1.00 & 1.03 & 3.74 & 3.13 \\
\hline p-xylene & 1.00 & 1.01 & 1.03 & 3.71 & 3.12 \\
\hline o-xylene & 0.92 & 0.94 & 0.96 & 3.86 & 3.12 \\
\hline 1-hexene & 2.68 & 2.62 & 2.57 & 1.91 & 3.07 \\
\hline 1-hexyne & 1.50 & 1.48 & 1.47 & 2.35 & 2.56 \\
\hline 1-heptyne & 1.81 & 1.83 & 1.83 & 2.77 & 3.21 \\
\hline 2-butanone & 0.39 & 0.37 & 0.34 & 3.06 & 0.34 \\
\hline 2-pentanone & 0.52 & 0.53 & 0.54 & 3.40 & 0.82 \\
\hline 3-pentanone & 0.47 & 0.48 & 0.50 & 3.41 & 0.91 \\
\hline 1,4-dioxane & 0.38 & 0.35 & 0.38 & 3.50 & -0.21 \\
\hline methanol & 1.61 & 1.32 & 1.36 & 2.24 & -1.50 \\
\hline ethanol & 1.46 & 1.34 & 1.22 & 2.59 & -1.08 \\
\hline 1-propanol & 1.62 & 1.47 & 1.32 & 2.98 & -0.58 \\
\hline 2-propanol & 1.52 & 1.38 & 1.25 & 2.69 & -0.79 \\
\hline 2-methyl-1-propanol & 1.70 & 1.53 & 1.39 & 3.21 & -0.09 \\
\hline 1-butanol & 2.20 & 1.85 & 1.64 & 3.28 & -0.18 \\
\hline diethyl ether & 1.27 & 1.25 & 1.25 & 1.80 & 0.63 \\
\hline diisopropyl ether & 2.29 & 2.28 & 2.26 & 2.09 & 1.04 \\
\hline chloroform & 0.39 & 0.39 & 0.41 & 2.79 & 2.00 \\
\hline dichloromethane & 0.27 & 0.30 & 0.30 & 2.49 & 1.53 \\
\hline tetrachloromethane & 1.32 & 1.12 & 1.31 & 2.48 & 2.67 \\
\hline acetonitrile & 0.49 & 0.50 & 0.49 & 2.99 & 0.14 \\
\hline nitromethane & 0.63 & 0.62 & 0.60 & 3.24 & 0.29 \\
\hline 1-nitropropane & 0.62 & 0.61 & 0.60 & 3.81 & 1.36 \\
\hline pyridine & 0.47 & 0.47 & 0.47 & 3.64 & 0.20 \\
\hline thiophene & 0.60 & 0.60 & 0.61 & 2.96 & 1.92 \\
\hline
\end{tabular}

$$
\begin{aligned}
\log K_{\mathrm{L}}= & \mathrm{c}_{\text {cation }}+\mathrm{c}_{\text {anion }}+\left(\mathrm{e}_{\text {cation }}+\mathrm{e}_{\text {anion }}\right) \mathbf{E} \\
& +\left(\mathrm{s}_{\text {cation }}+\mathrm{s}_{\text {anion }}\right) \mathbf{S}+\left(\mathrm{a}_{\text {cation }}+\mathrm{a}_{\text {anion }}\right) \mathbf{A} \\
& +\left(\mathrm{b}_{\text {cation }}+\mathrm{b}_{\text {anion }}\right) \mathbf{B}+\left(\mathrm{l}_{\text {cation }}+\mathrm{l}_{\text {anion }}\right) \mathbf{L}
\end{aligned}
$$

$$
\begin{aligned}
\log P= & \mathrm{c}_{\text {cation }}+\mathrm{c}_{\text {anion }}+\left(\mathrm{e}_{\text {cation }}+\mathrm{e}_{\text {anion }}\right) \mathbf{E} \\
& +\left(\mathrm{s}_{\text {cation }}+\mathrm{s}_{\text {anion }}\right) \mathbf{S}+\left(\mathrm{a}_{\text {cation }}+\mathrm{a}_{\text {anion }}\right) \mathbf{A} \\
& +\left(\mathrm{b}_{\text {cation }}+\mathrm{b}_{\text {anion }}\right) \mathbf{B}+\left(\mathrm{v}_{\text {cation }}+\mathrm{v}_{\text {anion }}\right) \mathbf{V}
\end{aligned}
$$

wherein each equation coefficient is split into a cation- and an anion-specific contribution. The experimental data measured

\begin{tabular}{|c|c|c|c|c|c|}
\hline \multirow[b]{2}{*}{ solutes } & \multicolumn{5}{|c|}{$T / K$} \\
\hline & 322.6 & 332.5 & 342.8 & $R^{2}$ & 298.2 \\
\hline hexane & 1.48 & 1.37 & 1.25 & 1.000 & 1.81 \\
\hline 3-methylpentane & 1.43 & 1.34 & 1.21 & 0.992 & 1.76 \\
\hline heptane & 1.85 & 1.70 & 1.56 & 1.000 & 2.24 \\
\hline octane & 2.20 & 2.04 & 1.88 & 1.000 & 2.66 \\
\hline nonane & 2.55 & 2.37 & 2.19 & 1.000 & 3.06 \\
\hline decane & 2.90 & 2.70 & 2.50 & 1.000 & 3.47 \\
\hline undecane & 3.25 & 3.02 & 2.80 & 1.000 & 3.88 \\
\hline methylcyclopentane & 1.66 & 1.54 & 1.43 & 1.000 & 1.98 \\
\hline cyclohexane & 1.80 & 1.68 & 1.56 & 1.000 & 2.15 \\
\hline methylcyclohexane & 2.02 & 1.88 & 1.75 & 1.000 & 2.40 \\
\hline cycloheptane & 2.30 & 2.16 & 2.01 & 1.000 & 2.71 \\
\hline benzene & 2.46 & 2.31 & 2.16 & 1.000 & 2.88 \\
\hline toluene & 2.83 & 2.66 & 2.50 & 1.000 & 3.31 \\
\hline ethylbenzene & 3.13 & 2.94 & 2.76 & 1.000 & 3.65 \\
\hline m-xylene & 3.20 & 3.01 & 2.82 & 1.000 & 3.74 \\
\hline p-xylene & 3.18 & 2.99 & 2.80 & 1.000 & 3.71 \\
\hline o-xylene & 3.32 & 3.12 & 2.93 & 1.000 & 3.86 \\
\hline 1-hexene & 1.57 & 1.45 & 1.33 & 1.000 & 1.91 \\
\hline 1-hexyne & 1.96 & 1.82 & 1.68 & 1.000 & 2.35 \\
\hline 1-heptyne & 2.32 & 2.16 & 2.00 & 1.000 & 2.77 \\
\hline 2-butanone & 2.62 & 2.46 & 2.31 & 1.000 & 3.06 \\
\hline 2-pentanone & 2.92 & 2.75 & 2.58 & 1.000 & 3.40 \\
\hline 3-pentanone & 2.92 & 2.75 & 2.58 & 1.000 & 3.41 \\
\hline 1,4-dioxane & 3.04 & 2.90 & 2.71 & 0.989 & 3.50 \\
\hline methanol & 1.86 & 1.78 & 1.61 & 0.961 & 2.24 \\
\hline ethanol & 2.18 & 2.03 & 1.89 & 1.000 & 2.59 \\
\hline 1-propanol & 2.52 & 2.36 & 2.20 & 1.000 & 2.98 \\
\hline 2-propanol & 2.26 & 2.11 & 1.96 & 1.000 & 2.69 \\
\hline 2-methyl-1-propanol & 2.72 & 2.54 & 2.37 & 1.000 & 3.21 \\
\hline 1-butanol & 2.82 & 2.66 & 2.49 & 1.000 & 3.28 \\
\hline diethyl ether & 1.48 & 1.37 & 1.25 & 1.000 & 1.80 \\
\hline diisopropyl ether & 1.72 & 1.58 & 1.46 & 1.000 & 2.09 \\
\hline chloroform & 2.38 & 2.24 & 2.09 & 1.000 & 2.79 \\
\hline dichloromethane & 2.13 & 1.97 & 1.87 & 0.986 & 2.49 \\
\hline tetrachloromethane & 2.07 & 2.00 & 1.80 & 0.928 & 2.48 \\
\hline acetonitrile & 2.59 & 2.44 & 2.30 & 0.999 & 2.99 \\
\hline nitromethane & 2.82 & 2.66 & 2.52 & 1.000 & 3.24 \\
\hline 1-nitropropane & 3.32 & 3.14 & 2.96 & 1.000 & 3.81 \\
\hline pyridine & 3.16 & 2.99 & 2.81 & 1.000 & 3.64 \\
\hline thiophene & 2.54 & 2.39 & 2.24 & 1.000 & 2.96 \\
\hline
\end{tabular}
here can be used to calculate the Abraham model ion-specific equation coefficients for the $[\mathrm{HMPyrr}]^{+},[\mathrm{OMPyrr}]^{+}$, and $[\mathrm{DMPyrr}]^{+}$cations for both the gas-to-IL (eq 8) and water-to-
Table 6. Logarithm of Partition Coefficients $\log K_{\mathrm{L}}$ of Organic Solutes in $[\mathrm{DMPyrr}]^{+}\left[\mathrm{Tf}_{2} \mathrm{~N}\right]^{-}$

Table 7. Comparison of Values of $\gamma^{\infty}$ in $[\mathrm{HMPyrr}]^{+}\left[\mathrm{Tf}_{2} \mathrm{~N}\right]^{-}$ and in $[\mathrm{OMPyrr}]^{+}\left[\mathrm{Tf}_{2} \mathrm{~N}\right]^{-}$Obtained in This Work with $\gamma^{\infty}$ Measured by Nebig et al. (ref 47$)$ at $T=323.15 \mathrm{~K}$

\begin{tabular}{lccccc} 
& \multicolumn{2}{c}{$[\mathrm{HMPyrr}]^{+}\left[\mathrm{Tf}_{2} \mathrm{~N}\right]^{-}$} & & \multicolumn{2}{c}{$[\mathrm{OMPyrr}]^{+}\left[\mathrm{Tf}_{2} \mathrm{~N}\right]^{-}$} \\
\cline { 2 - 3 } \cline { 5 - 6 } solutes & $\gamma^{\infty}$ (this work) & $\gamma^{\infty}($ ref 47$)$ & & $\gamma^{\infty}$ (this work) & $\gamma^{\infty}($ ref 47$)$ \\
hexane & 7.21 & 7.74 & & 4.90 & 5.01 \\
heptane & 10.26 & 10.58 & & 6.54 & 6.40 \\
octane & 13.93 & 14.25 & & 8.39 & 8.10 \\
1-hexene & 4.55 & 4.56 & & 3.22 & 3.17 \\
\hline
\end{tabular}

IL (eq 9) partitioning processes. The $\log P$ values for partition from water to the IL are calculated through eq 10

$$
\log P=\log K_{\mathrm{L}}-\log K_{\mathrm{W}}
$$

and require knowledge of the solute's gas phase partition coefficient into water $\left(K_{\mathrm{W}}\right)$ which is available for most of the 
Table 8. Selectivities $\left(S_{1,2}^{\infty}\right)$ and Capacities $\left(k_{1}^{\infty}\right)$ at Infinite Dilution for Different Separation Problems at $323.15 \mathrm{~K}$

\begin{tabular}{|c|c|c|c|c|c|c|}
\hline \multicolumn{2}{|r|}{ ILs } & \multirow[b]{2}{*}{ hexane/benzene } & \multirow[b]{2}{*}{ hexane/methanol } & \multirow[b]{2}{*}{ hexane/thiophene } & \multirow[b]{2}{*}{ cyclohexane/thiophene } & \multirow[b]{2}{*}{ ref } \\
\hline anion & cation & & & & & \\
\hline \multirow{24}{*}[\mathrm{Tf}_{2}\mathrm{N}]{$^{-}$} & 1-hexyl-1-methylpyrrolidinium & $10.2 / 1.32$ & $8.2 / 1.06$ & $11.1 / 1.43$ & $7.4 / 1.43$ & this work \\
\hline & 1-octyl-1-methylpyrrolidinium & $8.4 / 1.67$ & $5.2 / 1.03$ & $8.8 / 1.75$ & $6.1 / 1.75$ & this work \\
\hline & 1-decyl-1-methylpyrrolidinium & $6.4 / 1.61$ & $2.5 / 0.62$ & $6.6 / 1.67$ & $4.7 / 1.67$ & this work \\
\hline & 1-butyl-1-methylpyrrolidinium & $13.8 / 1.14$ & & & & 48 \\
\hline & methyltributylammonium & $7.5 / 1.11$ & $7.3 / 1.08$ & $7.8 / 1.17$ & $5.5 / 1.17$ & 49 \\
\hline & octyltrimethylammonium & $8.5 / 1.37$ & $6.75 / 1.08$ & $9 / 1.45$ & $5.9 / 1.45$ & 49 \\
\hline & decyltrimethylammonium & $5.9 / 1.41$ & $5.3 / 1.09$ & $6.46 / 1.54$ & $4.46 / 1.54$ & 49 \\
\hline & tetraoctylammonium & $3.2 / 2.5$ & $2.0 / 1.08$ & $3.25 / 2.5$ & $2.35 / 2.5$ & 49 \\
\hline & 1,3-dimethoxyimidazolium & $21.3 / 0.47$ & $42.05 / 0.94$ & $24.8 / 0.94$ & $12.6 / 0.94$ & 27 \\
\hline & 1-(methylethylether)-3-methylimidazolium & $15.5 / 0.85$ & $17.4 / 0.93$ & $18.1 / 1.0$ & $10.9 / 1.0$ & 27 \\
\hline & 1-ethanol-3-methylimidazolium & $20.6 / 0.47$ & $49.1 / 1.12$ & $24.7 / 0.56$ & $14.2 / 0.56$ & 27 \\
\hline & 1-ethyl-3-methylimidazolium & $37.5 / 1.43$ & $19.5 / 1.20$ & & & 50 \\
\hline & 1-(hexylmethylether)-3-methylimidazolium & $9.1 / 1.23$ & $6.8 / 0.91$ & $10.0 / 1.35$ & $6.4 / 1.35$ & 51 \\
\hline & 1,3-bis(hexylmethylether)imidazolium & $4.9 / 1.67$ & $3.2 / 1.06$ & $5.3 / 1.75$ & $3.7 / 1.75$ & 51 \\
\hline & 1-butyl-3-methylimidazolium & $16.7 / 1.11$ & & & & 52 \\
\hline & 1-hexyl-3-methylimidazolium & $9.5 / 1.29$ & $6.1 / 0.82$ & & & 53 \\
\hline & trimethylhexylammonium & $9.9 / 1.01$ & $8.5 / 0.86$ & $10.7 / 1.09$ & $7.2 / 1.09$ & 26 \\
\hline & 4-methyl- $N$-butylpyridinium & $18.8 / 1.43$ & $21.2 / 0.83$ & $10.6 / 1.56$ & $6.1 / 1.56$ & 54 \\
\hline & triethylsulfonium & $21.6 / 0.91$ & $17.8 / 0.77$ & $25.5 / 1.05$ & $14.3 / 1.05$ & 55 \\
\hline & trihexyl(tetradecyl)phosphonium & $2.7 / 2.56$ & $1.1 / 1.02$ & $2.6 / 2.5$ & $1.95 / 2.5$ & 28 \\
\hline & 1-(2-methoxyethyl)-1-methylpiperidinium & $17.9 / 1.09$ & $12.5 / 0.76$ & $20.7 / 1.26$ & $12.0 / 1.26$ & 56 \\
\hline & 4-(2-methoxyethyl)-4-methylmorpholinium & $25.8 / 0.75$ & $27.9 / 0.81$ & $31.7 / 0.92$ & $16.5 / 0.92$ & 57 \\
\hline & 1-propyl-1-methylpiperidinium & $19.8 / 1.07$ & $12.8 / 0.69$ & $22.6 / 1.22$ & $13.1 / 1.22$ & 58 \\
\hline & 1-butyl-1-methylpiperidinium & $15.4 / 1.27$ & $8.2 / 0.68$ & $16.8 / 1.39$ & $10.4 / 1.39$ & 59 \\
\hline
\end{tabular}

solutes studied. ${ }^{30}$ Water-to-IL partition coefficients calculated through eq 8 pertain to a hypothetical partitioning process involving solute transfer from water to the anhydrous IL. $\log P$ values calculated in this manner are still quite useful in that predicted $\log P$ values can be used to estimate the solute's infinite dilution activity coefficient in the IL. For convenience, we have captured in Tables 1,3 , and 5 the $\log P$ and $\log K_{\mathrm{L}}$ values at $298.15 \mathrm{~K}$ for anhydrous $[\mathrm{HMPyrr}]^{+}\left[\mathrm{Tf}_{2} \mathrm{~N}\right]^{-}$, $[\mathrm{OMPyrr}]^{+}\left[\mathrm{Tf}_{2} \mathrm{~N}\right]^{-}$, and $[\mathrm{DMPyrr}]^{+}\left[\mathrm{Tf}_{2} \mathrm{~N}\right]^{-}$, respectively.

Analysis of the 36 experimental $\log K_{\mathrm{L}}$ and $\log P$ data in Table 1 for $[\mathrm{HMPyrr}]^{+}\left[\mathrm{Tf}_{2} \mathrm{~N}\right]^{-}$gave

$$
\begin{aligned}
\log K_{\mathrm{L}}= & -0.533(0.093)-0.110(0.099) \mathbf{E} \\
& +2.146(0.095) \mathbf{S}+2.278(0.196) \mathbf{A} \\
& +0.650(0.114) \mathbf{B}+0.767(0.026) \mathbf{L}
\end{aligned}
$$

$$
\left(N=36, \mathrm{SD}=0.088, R^{2}=0.984, F=367.3\right)
$$

and

$$
\begin{aligned}
& \log P=-0.226(0.152)-0.083(0.132) \mathbf{E}+0.560(0.144) \mathbf{S} \\
&-1.301(0.275) \mathbf{A}-4.501(0.159) \mathbf{B} \\
&+3.673(0.128) \mathbf{V} \\
&\left(N=36, \mathrm{SD}=0.123, R^{2}=0.994, F=1098\right)
\end{aligned}
$$

where $N$ denotes the number of experimental values used in the regression analysis, $\mathrm{SD}$ refers to the standard deviation, $R^{2}$ is the squared correlation coefficient, and $F$ corresponds to the Fisher F-statistic. The standard errors in the calculated coefficients are given in parentheses immediately following the respective values. The solute descriptors used in the analysis are listed in Table 9. As an informational note 1,4-dioxane was excluded from the regression analyses because of uncertainties associated in extrapolating the measured $\log K_{\mathrm{L}}$ data back to $298.15 \mathrm{~K}$. The squared correlation coefficients for the $\log K_{\mathrm{L}}$ versus $T^{-1}$ curves for this one solute was much smaller than unity, as shown in fifth column of Table 2.

Each calculated equation coefficient corresponds to the sum of the respective cation-specific and anion-specific contributions. In establishing this computation methodology, the equation coefficients for the $\left[\mathrm{Tf}_{2} \mathrm{~N}\right]^{-}$anion were set equal to zero to provide a reference point from which all equation coefficients would be calculated. ${ }^{38-41}$ A common reference point is needed because the cation-specific and anion-specific equation coefficients are generated as a paired set. The equation coefficients in eqs 11 and 12 thus correspond to the ion-specific coefficients for the $[\mathrm{HMPyrr}]^{+}$cation.

The experimental $\log K_{\mathrm{L}}$ and $\log P$ data in Tables 3 and 5 for $[\mathrm{OMPyrr}]^{+}\left[\mathrm{Tf}_{2} \mathrm{~N}\right]^{-}$and $[\mathrm{DMPyrr}]^{+}\left[\mathrm{Tf}_{2} \mathrm{~N}\right]^{-}$were analyzed in a similar manner, to give the following Abraham model correlations:

$$
\begin{aligned}
& \log K_{\mathrm{L}}=-0.587(0.082)-0.064(0.090) \mathbf{E} \\
&+2.080(0.086) \mathbf{S}+2.176(0.160) \mathbf{A} \\
&+0.486(0.103) \mathbf{B}+0.822(0.023) \mathbf{L} \\
&\left(N=37, \mathrm{SD}=0.080, R^{2}=0.986, F=449.1\right) \\
& \log P=-0.253(0.121)+0.520(0.096) \mathbf{S} \\
& \quad-1.460(0.203) \mathbf{A}-4.696(0.116) \mathbf{B} \\
& \quad+3.815(0.103) \mathbf{V} \\
&\left(N=37, \mathrm{SD}=0.102, R^{2}=0.997, F=2372\right)
\end{aligned}
$$


Table 9. Solute Descriptors of Organic Compounds Considered in this Study

\begin{tabular}{|c|c|c|c|c|c|c|}
\hline solute & $\mathbf{E}$ & $S$ & A & B & $\mathbf{L}$ & $\mathbf{V}$ \\
\hline hexane & 0.000 & 0.000 & 0.000 & 0.000 & 2.668 & 0.9540 \\
\hline 3-methylpentane & 0.000 & 0.000 & 0.000 & 0.000 & 2.581 & 0.9540 \\
\hline heptane & 0.000 & 0.000 & 0.000 & 0.000 & 3.173 & 1.0949 \\
\hline octane & 0.000 & 0.000 & 0.000 & 0.000 & 3.677 & 1.2358 \\
\hline nonane & 0.000 & 0.000 & 0.000 & 0.000 & 4.182 & 1.3767 \\
\hline decane & 0.000 & 0.000 & 0.000 & 0.000 & 4.686 & 1.5176 \\
\hline undecane & 0.000 & 0.000 & 0.000 & 0.000 & 5.191 & 1.6590 \\
\hline methylcyclopentane & 0.225 & 0.100 & 0.000 & 0.000 & 2.907 & 0.8454 \\
\hline cyclohexane & 0.310 & 0.100 & 0.000 & 0.000 & 2.964 & 0.8454 \\
\hline methylcyclohexane & 0.244 & 0.060 & 0.000 & 0.000 & 3.319 & 0.9863 \\
\hline cycloheptane & 0.350 & 0.100 & 0.000 & 0.000 & 3.704 & 0.9863 \\
\hline benzene & 0.610 & 0.520 & 0.000 & 0.140 & 2.786 & 0.7164 \\
\hline toluene & 0.601 & 0.520 & 0.000 & 0.140 & 3.325 & 0.8573 \\
\hline ethylbenzene & 0.613 & 0.510 & 0.000 & 0.150 & 3.778 & 0.9982 \\
\hline$m$-xylene & 0.623 & 0.520 & 0.000 & 0.160 & 3.839 & 0.9982 \\
\hline$p$-xylene & 0.613 & 0.520 & 0.000 & 0.160 & 3.839 & 0.9982 \\
\hline$o$-xylene & 0.663 & 0.560 & 0.000 & 0.160 & 3.939 & 0.9982 \\
\hline 1-hexene & 0.080 & 0.080 & 0.000 & 0.070 & 2.572 & 0.9110 \\
\hline 1-hexyne & 0.166 & 0.220 & 0.100 & 0.120 & 2.510 & 0.8680 \\
\hline 1-heptyne & 0.160 & 0.230 & 0.090 & 0.100 & 3.000 & 1.0089 \\
\hline 2-butanone & 0.166 & 0.700 & 0.000 & 0.510 & 2.287 & 0.6879 \\
\hline 2-pentanone & 0.143 & 0.680 & 0.000 & 0.510 & 2.755 & 0.8288 \\
\hline 3-pentanone & 0.154 & 0.660 & 0.000 & 0.510 & 2.811 & 0.8288 \\
\hline 1,4-dioxane & 0.329 & 0.750 & 0.000 & 0.640 & 2.892 & 0.6810 \\
\hline methanol & 0.278 & 0.440 & 0.430 & 0.470 & 0.970 & 0.3082 \\
\hline ethanol & 0.246 & 0.420 & 0.370 & 0.480 & 1.485 & 0.4491 \\
\hline 1-propanol & 0.236 & 0.420 & 0.370 & 0.480 & 2.031 & 0.5900 \\
\hline 2-propanol & 0.212 & 0.360 & 0.330 & 0.560 & 1.764 & 0.5900 \\
\hline 2-methyl-1-propanol & 0.217 & 0.390 & 0.370 & 0.480 & 2.413 & 0.7309 \\
\hline 1-butanol & 0.224 & 0.420 & 0.370 & 0.480 & 2.601 & 0.7309 \\
\hline diethyl ether & 0.041 & 0.250 & 0.000 & 0.450 & 2.015 & 0.7309 \\
\hline diisopropyl ether & -0.063 & 0.170 & 0.000 & 0.570 & 2.501 & 1.0127 \\
\hline chloroform & 0.425 & 0.490 & 0.150 & 0.020 & 2.480 & 0.6167 \\
\hline dichloromethane & 0.390 & 0.570 & 0.100 & 0.050 & 2.019 & 0.4943 \\
\hline tetrachloromethane & 0.460 & 0.380 & 0.000 & 0.000 & 2.823 & 0.7391 \\
\hline acetonitrile & 0.237 & 0.900 & 0.070 & 0.320 & 1.739 & 0.4042 \\
\hline nitromethane & 0.313 & 0.950 & 0.060 & 0.310 & 1.892 & 0.4237 \\
\hline 1-nitropropane & 0.242 & 0.950 & 0.000 & 0.310 & 2.894 & 0.7055 \\
\hline pyridine & 0.631 & 0.840 & 0.000 & 0.520 & 3.022 & 0.6753 \\
\hline thiophene & 0.687 & 0.570 & 0.000 & 0.150 & 2.819 & 0.6411 \\
\hline
\end{tabular}

$$
\begin{aligned}
\log K_{\mathrm{L}}= & -0.395(0.062) \mathbf{E}-0.241(0.070) \mathbf{E} \\
& +1.991(0.067) \mathbf{S}+2.112(0.110) \mathbf{A} \\
& +0.268(0.076) \mathbf{B}+0.822(0.018) \mathbf{L}
\end{aligned}
$$

$$
\left(N=40, \mathrm{SD}=0.063, R^{2}=0.990, F=682\right)
$$

$$
\begin{aligned}
\log P= & -0.083(0.128)-0.142(0.114) \mathbf{E}+0.419(0.124) \mathbf{S} \\
& -1.467(0.197) \mathbf{A}-4.859(0.129) \mathbf{B} \\
& +3.824(0.108) \mathbf{V}
\end{aligned}
$$

$$
\left(N=40, \mathrm{SD}=0.108, R^{2}=0.997, F=2167\right)
$$

Equations 13 and 14 pertain to solute transfer to anhydrous $[\mathrm{OMPyrr}]^{+}\left[\mathrm{Tf}_{2} \mathrm{~N}\right]^{-}$, and eqs 15 and 16 in turn describe the partition coefficient data for solutes dissolved in $[\mathrm{DMPyrr}]^{+}\left[\mathrm{Tf}_{2} \mathrm{~N}\right]^{-}$. The eE term was found to be negligible in the $\log P$ correlation $(\mathrm{e}=0.023)$ for $[\mathrm{OMPyrr}]^{+}\left[\mathrm{Tf}_{2} \mathrm{~N}\right]^{-}$, so it was eliminated from the final correlation. Methanol and 1,4dioxane were removed from the $[\mathrm{OMPyrr}]^{+}\left[\mathrm{Tf}_{2} \mathrm{~N}\right]^{-}$data sets because of low squared correlation coefficients in the $\log K_{\mathrm{L}}$ versus $T^{-1}$ plots used to extrapolate the measured partition coefficient data back to $298.15 \mathrm{~K}$. The derived Abraham model correlations provide a good mathematical description of the experimental gas-to-anhydrous IL partition coefficient data (eqs 13 and 15) and water-to-anhydrous IL partition coefficient data (eqs 14 and 16) for both $[\mathrm{OMPyrr}]^{+}\left[\mathrm{Tf}_{2} \mathrm{~N}\right]^{-}$and $[\mathrm{DMPyrr}]^{+}\left[\mathrm{Tf}_{2} \mathrm{~N}\right]^{-}$. Since both ILs contain the $\left[\mathrm{Tf}_{2} \mathrm{~N}\right]^{-}$ anion, the calculated coefficients in eqs 13-16 thus correspond to the cation-specific coefficients for $[\mathrm{OMPyrr}]^{+}$and $[\mathrm{DMPyrr}]^{+}$.

\section{CONCLUSION}

The Abraham model correlations that have been derived for $[\mathrm{HMPyrr}]^{+}\left[\mathrm{Tf}_{2} \mathrm{~N}\right]^{-}, \quad[\mathrm{OMPyrr}]^{+}\left[\mathrm{Tf}_{2} \mathrm{~N}\right]^{-}$, and $[\mathrm{DMPyrr}]^{+}\left[\mathrm{Tf}_{2} \mathrm{~N}\right]^{-}$would be expected to provide very reasonable predictions for the $\log K_{\mathrm{L}}$ values for additional 
solutes having descriptor values that fall within the range of chemical space defined by $\mathbf{E}=-0.063$ to $+0.687, \mathbf{S}=0.000$ to $0.950, \mathrm{~A}=0.000$ to $0.570, \mathrm{~B}=0.000$ to $0.640, \mathrm{~L}=0.970$ to 5.191, and $\mathbf{V}=0.308$ to 1.659 . The cation-specific and anionspecific equation coefficients can be combined to yield predictive equations for numerous ILs not yet studied. Specifically, the present study expands our library of calculated cation-specific equation coefficients to 27 which, when combined with our hitherto reported equation coefficients for 14 unique anions, yield predictive equations for 378 different ILs. Once a substantial inventory of ion-specific coefficients has been established, it is easy to see how adding a handful of cation- and/or anion-specific equation coefficients can quickly enlarge the databank of IL permutations which can be predicted within such an Abraham solvation parameter framework. Indeed, expanding this chemical space forms a significant portion of our current research efforts.

\section{AUTHOR INFORMATION}

\section{Corresponding Author}

*E-mail: acree@unt.edu.

\section{Notes}

The authors declare no competing financial interest.

\section{REFERENCES}

(1) Asumana, C.; Yu, G.; Guan, Y.; Yang, S.; Zhou, S.; Chen, X. Extractive Denitrogenation of Fuel Oils with Dicyanamide-based Ionic Liquids. Green Chem. 2011, 13, 3300-3305.

(2) Anantharaj, R.; Banerjee, T. COSMO-RS-Based Screening of Ionic Liquids as Green Solvents in Denitrification Studies. Ind. Eng. Chem. Res. 2010, 49, 8705-8725.

(3) Mochizuki, Y.; Sugawara, K. Removal of Organic Sulfur from Hydrocarbon Resources using Ionic Liquids. Energy Fuels 2008, 22, 3303-3307.

(4) Kumar, A. A. P.; Banerjee, T. Thiophene Separation with Ionic Liquids for Desulphurization: A Quantum Chemical Approach. Fluid Phase Equilib. 2009, 278, 1-8.

(5) Gao, H.; Luo, M.; Xing, J.; Wu, Y.; Li, Y.; Li, W.; Liu, Q.; Liu, H. Desulfurization of Fuel by Extraction with Pyridinium-based Ionic Liquids. Ind. Eng. Chem. Res. 2008, 47, 8384-8388.

(6) Nie, Y.; Li, C.; Meng, H.; Wang, Z. N,N-Dialkylimidazolium Dialkylphosphate Ionic Liquids: Their Extractive Performance for Thiophene Series Compounds from Fuel Oils Versus the Length of Alkyl Group. Fuel Process. Technol. 2008, 89, 978-983.

(7) Pereiro, A. B.; Araujo, J. M. M.; Esperanca, J. M. S. S.; Marrucho, I. M.; Rebelo, L. P. N. Ionic Liquids in Separations of Azeotropic Systems-A Review. J. Chem. Thermodyn. 2012, 46, 2-28.

(8) Zhao, X.; Xing, H.; Yang, Q.; Li, R.; Su, B.; Bao, Z.; Yang, Y.; Ren, Q. Differential Solubility of Ethylene and Acetylene in Roomtemperature Ionic Liquids: A Theoretical Study. J. Phys. Chem. B 2012, 116, 3944-3953.

(9) Palgunadi, J.; Hong, S. Y.; Lee, J. K.; Lee, H.; Lee, S. D.; Cheong, M.; Kim, H. S. Correlation between Hydrogen Bond Basicity and Acetylene Solubility in Room Temperature Ionic Liquids. J. Phys. Chem. B 2011, 115, 1067-1074.

(10) Palgunadi, J.; Indarto, A. Room Temperature Ionic Liquids for Propyne-propylene Separations: Solubility Behaviors and Selectivity Investigation. Chem. Eng. Sci. 2011, 66, 6039-6047.

(11) Mokrushin, V.; Assenbaum, D.; Paape, N.; Gerhard, D.; Mokrushina, L.; Wasserscheid, P.; Arlt, W.; Kistenmacher, H.; Neuendorf, S.; Goeke, V. Ionic Liquids for Propene-propane Separation. Chem. Eng. Technol. 2010, 33, 63-73.

(12) Ortiz, A.; Galan, L. M.; Gorri, D.; de Haan, A. B.; Ortiz, I. Reactive Ionic Liquid Media for the Separation of Propylene/propane Gaseous Mixtures. Ind. Eng. Chem. Res. 2010, 49, 7227-7233.
(13) Lei, Z.; Arlt, W.; Wasserscheid, P. Separation of 1-Hexene and n-Hexane with Ionic Liquids. Fluid Phase Equilib. 2006, 241, 290-299.

(14) Scovazzo, P. Determination of the Upper Limits, Benchmarks, and Critical Properties for Gas Separations using Stabilized Room Temperature Ionic Liquid Membranes (SILMs) for the Purpose of Guiding Future Research. J. Membr. Sci. 2009, 343, 199-211.

(15) Gin, D. L.; Noble, R. D.; Carlisle, T. K.; Voss, B. A.; Bara, J. E.; LaFrate, A. L.; Miller, A. L., II; Hudiono, Y. C.; Wiesenauer, B. R.; Reynolds, M. E. New Functionalized Imidazolium-based Roomtemperature Ionic Liquids and Composite Materials for Gas Separation and Selective Transport Applications. ECS Trans. 2010, $33,21-34$

(16) Tang, S.; Baker, G. A.; Zhao, H. Ether- and Alcoholfunctionalized Task-specific Ionic Liquids: Attractive Properties and Applications. Chem. Soc. Rev. 2012, 41, 4030-4066.

(17) Abraham, M. H. Scales of Solute Hydrogen-bonding: Their Construction and Application to Physicochemical and Biochemical Processes. Chem. Soc. Rev. 1993, 22, 73-83.

(18) Abraham, M. H.; Ibrahim, A.; Zissimos, A. M. Determination of Sets of Solute Descriptors From Chromatographic Measurements. J. Chromatogr. A 2004, 1037, 29-47.

(19) Abraham, M. H.; Grellier, P. L.; Mc Gill, R. A. Determination of Olive Oil-gas and Hexadecane-gas Partition Coefficients, and Calculation of the Corresponding Olive Oil-water and Hexadecanewater Partition Coefficients. J. Chem. Soc., Perkin Trans. 2 1987, 797803.

(20) Abraham, M. H.; Whiting, G. S.; Doherty, R. M. Hydrogen Bonding. Part 13. A New Method for the Characterization of GLC Stationary Phases: The Lafford Data Set. J. Chem. Soc., Perkin Trans. 2 1990, 1451-1460.

(21) Abraham, M. H.; Whiting, G. S.; Doherty, R. M.; Shuely, W. J. Hydrogen Bonding XVI. A New Solute Solvation Parameter, $\pi_{2}{ }^{\mathrm{H}}$, from Gas Chromatographic Data. J. Chromatogr. 1991, 587, 213-228.

(22) Abraham, M. H.; Platts, J. A. Hydrogen Bond Structural Group Constants. J. Org. Chem. 2001, 66, 3484-3491.

(23) Platts, J. A.; Butina, D.; Abraham, M. H.; Hersey, A. Estimation of Molecular Linear Free Energy Relation Descriptors using a Group Contribution Approach. J. Chem. Inf. Comp. Sci. 1999, 39, 835-845.

(24) Mutelet, F.; Rogalski, M. Experimental Determination and Prediction of the Gas-liquid $n$-Hexadecane Partition Coefficients. J. Chromatogr. A 2001, 923, 153-163.

(25) Moise, J.-C.; Mutelet, F.; Jaubert, J.-N.; Grubbs, L. M.; Acree, W. E., Jr.; Baker, G. A. Activity Coefficients at Infinite Dilution of Organic Compounds in Four New Imidazolium-Based Ionic Liquids. J. Chem. Eng. Data 2011, 56, 3106-3114.

(26) Mutelet, F.; Revelli, A.-L.; Jaubert, J.-N.; Sprunger, L. M.; Acree, W. E., Jr.; Baker, G. A. Partition Coefficients of Organic Compounds in New Imidazolium and Tetralkylammonium Based Ionic Liquids using Inverse Gas Chromatography. J. Chem. Eng. Data 2010, 55, 234-242.

(27) Revelli, A.-L.; Mutelet, F.; Jaubert, J.-N.; Garcia-Martinez, M.; Sprunger, L. M.; Acree, W. E., Jr.; Baker, G. A. Study of Ether-, Alcohol-, or Cyano-functionalized Ionic Liquids using Inverse Gas Chromatography. J. Chem. Eng. Data 2010, 55, 2434-2443.

(28) Revelli, A.-L.; Sprunger, L. M.; Gibbs, J.; Acree, W. E., Jr.; Baker, G. A.; Mutelet, F. Activity Coefficients at Infinite Dilution of Organic Compounds in Trihexyl(tetradecyl)-phosphonium Bis(trifluoromethylsulfonyl)imide using Inverse Gas Chromatography. J. Chem. Eng. Data 2009, 54, 977-985.

(29) Acree, W. E., Jr.; Abraham, M. H. The Analysis of Solvation in Ionic Liquids and Organic Solvents using the Abraham Linear Free Energy Relationship. J. Chem. Technol. Biotechnol. 2006, 81, 14411446. [Erratum: J. Chem. Technol. Biotechnol. 2006, 81, 1722]

(30) Abraham, M. H.; Acree, W. E., Jr. Comparative Analysis of Solvation and Selectivity in Room Temperature Ionic Liquids using the Abraham Linear Free Energy Relationship. Green Chem 2006, 8, 906-915.

(31) Grubbs, L. M.; Ye, S.; Saifullah, M.; Acree, W. E., Jr.; Twu, P.; Anderson, J. L.; Baker, G. A.; Abraham, M. H. Correlation of the 
Solubilizing Abilities of Hexyl(trimethyl)-ammonium Bis(trifluoromethylsulfonyl)imide, 1-Propyl-1-methyl-piperidinium Bis(trifluoromethylsulfonyl)imide and 1-Butyl-1-methyl-pyrrolidinium Thiocyanate. J. Solution Chem. 2011, 40, 2000-2022.

(32) Sprunger, L. M.; Gibbs, J.; Baltazar, Q. Q.; Acree, W. E., Jr.; Abraham, M. H.; Anderson, J. L. Characterization of Room Temperature Ionic Liquid Chromatographic Stationary Phases by Combining Experimental Retention Factor and Partition Coefficient Data into a Single Model. Phys. Chem. Liq. 2009, 47, 74-83.

(33) Sprunger, L. M.; Acree, W. E., Jr.; Abraham, M. H. Linear Free Energy Relationship (LFER) Correlations for the Solubilising Characterization of Room Temperature Ionic Liquids Containing Triethylsulphonium and 1-Butyl-1-methylpyrrolidinium Cations. Phys. Chem. Liq. 2010, 48, 385-393.

(34) Proctor, A.; Sprunger, L. M.; Acree, W. E., Jr.; Abraham, M. H. LFER Correlations for the Solubilising Characterisation of Room Temperature Ionic Liquids Containing Trifluoromethanesulfonate and Trifluoroacetate anions. Phys. Chem. Liq. 2008, 46, 631-642.

(35) Stephens, T. W.; Acree, W. E., Jr.; Twu, P.; Anderson, J. L.; Baker, G. A.; Abraham, M. H. Correlation of the Solubilizing Abilities of 1-Butyl-1-methyl-piperidinium Bis(trifluoromethylsulfonyl)imide and 1-Butyl-1-methyl-pyrrolidinium Tetracyanoborate. J. Solution Chem. 2012, in press.

(36) Acree, W. E. Jr.; Grubbs, L. M.; Abraham, M. H. Selection of Ionic Liquid Solvents for Chemical Separations Based on the Abraham Model. In Ionic Liquids, Applications and Perspectives; Kokorin, A., Ed.; INTECH Publishers: Rijeka, Croatia, 2011; Book 2, Chapter 13, pp 273-302.

(37) Mutelet, F.; Moise, J.-C.; Skrzypczak, A. Evaluation of the Performance of Trigeminal Tricationic Ionic Liquids for Separation Problems. J. Chem. Eng. Data 2012, 57, 918-927.

(38) Sprunger, L.; Clark, M.; Acree, W. E., Jr.; Abraham, M. H. Characterization of Room-temperature Ionic Liquids by the Abraham model with Cation-specific and Anion-specific Equation Coefficients. J. Chem. Inf. Model. 2007, 47, 1123-1129.

(39) Sprunger, L. M.; Proctor, A.; Acree, W. E., Jr.; Abraham, M. H. LFER Correlations for Room Temperature Ionic Liquids: Separation of Equation Coefficients into Individual Cation-specific and Anionspecific Contributions. Fluid Phase Equilib. 2008, 265, 104-111.

(40) Sprunger, L. M.; Gibbs, J.; Proctor, A.; Acree, W. E., Jr.; Abraham, M. H.; Meng, Y.; Yao, C.; Anderson, J. L. Linear Free Energy Relationship Correlations for Room Temperature Ionic Liquids: Revised Cation-specific and Anion-specific Equation Coefficients for Predictive Applications Covering a Much Larger Area of Chemical Space. Ind. Eng. Chem. Res. 2009, 48, 4145-4154.

(41) Grubbs, L. M.; Saifullah, M.; De La Rosa, N. E.; Acree, W. E., Jr.; Abraham, M. H.; Zhao, Q.; Anderson, J. L. Cation-specific and Anion-specific Abraham Model Correlations for Solute Transfer into Ionic Liquid Solvents. Glob. J. Phys. Chem. 2010, 1, 1-19.

(42) Mutelet, F.; Ortega-Villa, V.; Moise, J.-C.; Jaubert, J.-N.; Acree, W. E., Jr. Prediction of Partition Coefficients of Organic Compounds in Ionic Liquids using a Temperature-dependent Linear Solvation Energy Relationship with Parameters Calculated through a Group Contribution Method. J. Chem. Eng. Data 2011, 56, 3598-3606.

(43) Jin, H.; O’Hare, B.; Dong, J.; Arzhantsev, S.; Baker, G. A.; Wishart, J. F.; Benesi, A. J.; Maroncelli, M. Physical Properties of Ionic Liquids Consisting of the 1-Butyl-3-methylimidazolium Cation with Various Anions and the Bis(trifluoromethylsulfonyl)imide Anion with Various Cations. J. Phys. Chem. B 2008, 112, 81-92.

(44) Burrell, A. K.; Del Sesto, R. E.; Baker, S. N.; McCleskey, T. M.; Baker, G. A. The Large Scale Synthesis of Pure Imidazolium and Pyrrolidinium Ionic Liquids. Green Chem. 2007, 9, 449-454.

(45) Pandey, S.; Baker, S. N.; Pandey, S.; Baker, G. A. Fluorescent Probe Studies of Polarity and Solvation within Room Temperature Ionic Liquids: A Review. J. Fluoresc. 2012, DOI: 10.1007/s10895-0121073-x.

(46) Cruickshank, A. J. B.; Windsor, M. L.; Young, C. L. The Use of Gas-liquid Chromatography to Determine Activity Coefficients and
Second Virial Coefficients of Mixtures. Proc. R. Soc. London 1966, A295, 259-270.

(47) Nebig, S.; Liebert, V.; Gmehling, J. Measurement and Prediction of Activity Coefficients at Infinite Dilution $\left(\gamma^{\infty}\right)$, Vapor-liquid Equilibria (VLE) and Excess Enthalpies $\left(\mathrm{H}^{\mathrm{E}}\right)$ of Binary Systems with 1,1-dialkyl-pyrrolidinium Bis(trifluoromethylsulfonyl)imide using mod. UNIFAC (Dortmund). Fluid Phase Equilib. 2009, 277, 61-67.

(48) Kato, R.; Gmehling, J. Systems with Ionic Liquids: Measurement of VLE and $\gamma^{\infty}$ Data and Prediction of their Thermodynamic Behavior using Original UNIFAC, mod. UNIFAC (Do) and COSMORS (Ol). J. Chem. Thermodyn. 2005, 37, 603-619.

(49) Acree, W. E., Jr.; Baker, G. A.; Mutelet, F.; Moise, J.-C. Partition Coefficients of Organic Compounds in Four New Tetraalkylammonium Bis(trifluoromethylsulfonyl)imide Ionic Liquids using Inverse Gas Chromatography. J. Chem. Eng. Data 2011, 56, 3688-3697.

(50) Deenadayalu, N.; Letcher, T. M.; Reddy, P. Determination of Activity Coefficients at Infinite Dilution of Polar and Nonpolar Solutes in the Ionic Liquid 1-Ethyl-3-methylimidazolium Bis(trifluoromethylsulfonyl)imidate using Gas-liquid Chromatography at the Temperature 303.15 or 318.15 K. J. Chem. Eng. Data 2005, 50, 105-108.

(51) Domanska, U.; Marciniak, A. Activity Coefficients at Infinite Dilution Measurements for Organic Solutes and Water in the 1Hexyloxymethyl-3-methyl-imidazolium and 1,3-Dihexyloxymethylimidazolium Bis(trifluoromethylsulfonyl)imide Ionic liquids. The Cation Influence. Fluid Phase Equilib. 2009, 286, 154-161.

(52) Krummen, M.; Wasserscheid, P.; Gmehling, J. Measurement of Activity Coefficients at Infinite Dilution in Ionic Liquids using the Dilutor Technique. J. Chem. Eng. Data 2002, 7, 1411-1417.

(53) Letcher, T. M.; Marciniak, A.; Marciniak, M.; Domanska, U. Activity Coefficients at Infinite Dilution Measurements for Organic Solutes in the Ionic Liquid 1-Hexyl-3-methyl-imidazolium Bis(trifluoromethylsulfonyl)imide using G.L.C. at $\mathrm{T}=(298.15,313.15$, and 333.15) K. J. Chem. Thermodyn. 2005, 37, 1327-1331.

(54) Domanska, U.; Marciniak, A. Activity Coefficients at Infinite Dilution Measurements for Organic Solutes and Water in the Ionic Liquid 4-Methyl-N-butyl-pyridinium Bis(trifluoromethylsulfonyl)imide. J. Chem. Thermodyn. 2009, 41, 1350-1355.

(55) Domanska, U.; Marciniak, A. Activity Coefficients at Infinite Dilution Measurements for Organic Solutes and Water in the Ionic Liquid Triethylsulphonium Bis(trifluoromethylsulfonyl)imide. J. Chem. Thermodyn. 2009, 41, 754-758.

(56) Marciniak, A.; Wlazlo, M. Activity Coefficients at Infinite Dilution and Physicochemical Properties for Organic Solutes and Water in the Ionic Liquid 1-(2-Methoxyethyl)-1-methylpiperidinium Bis(trifluoromethylsulfonyl)amide. J. Chem. Thermodyn. 2012, 49, $137-145$.

(57) Marciniak, A.; Wlazlo, M. Activity Coefficients at Infinite Dilution and Physicochemical Properties for Organic Solutes and Water in the Ionic Liquid 4-(2-Methoxyethyl)-4-methylmorpholinium Bis(trifluoromethylsulfonyl)amide. J. Chem. Thermodyn. 2012, 47, 382-388.

(58) Domanska, U.; Paduszynski, K. Measurements of Activity Coefficients at Infinite Dilution of Organic Solutes and Water in 1Propyl-1-methylpiperidinium Bis\{(trifluoromethyl)-sulfonyl $\}$ imide ionic liquid using G.L.C. J. Chem. Thermodyn. 2010, 42, 1361-1366.

(59) Paduszynski, K.; Domanska, U. Limiting Activity Coefficients and Gas-liquid Partition Coefficients of Various Solutes in Piperidinium Ionic Liquids: Measurements and LSER Calculations. J. Phys. Chem. B 2011, 115, 8207-8215. 